\title{
A Robust Region Control Approach for Simultaneous Trajectory Tracking and Physical Human-Robot Interaction
}

\author{
Xiangyun Li, Qi Lu, Member, IEEE, Jiali Chen and Kang Li, Member, IEEE
}

\begin{abstract}
In this work, the uncertainty and disturbance estimator (UDE)-based robust region tracking controller for a robot manipulator is developed to achieve the moving target region trajectory tracking and the compliant human-robot interaction simultaneously. Utilizing the back-stepping control approach, the UDE is seamlessly fused into the region tracking control framework to estimate and compensate the model uncertainty and external disturbance, such as unknown payload, unmodeled joint coupling effect and friction. The regional feedback error is derived from the potential function to drive the robot manipulator end-effector converging into the target region, where the robot manipulator can be passively manipulated based on the needs of human to achieve the compliant physical human-robot interaction. Extensive experimental studies are carried out with a universal robots 10 manipulator to validate the effectiveness of the proposed method for moving region trajectory tracking, handling unknown payload and compliant physical human-robot interaction. The superior robustness of the proposed approach is demonstrated by comparison with the existing controller under the adverse effect of unknown payload. The humanrobot interaction is achieved in a shared autonomy manner with the cooperation of the manipulator and the human subject to accomplish the temperature measurement task, where the variation in human-subject height and the complexity of aiming the thermometer are successfully accommodated.
\end{abstract}

Index Terms-region tracking control, uncertainty and disturbance estimator, robot manipulator, human-robot interaction.

\section{INTRODUCTION}

$\mathbf{T}$ HE outbreak of the novel corona-virus disease 2019 (COVID-19) which features high contagiousness and infection fatality rate has now become a once-in-a-century pandemic [1]. At the time of writing, this pandemic has affected more than 200 countries and territories around the world with more than 207 million confirmed cases [2]. Healthcare workers, like nurses and doctors, are in direct contact with the patients who carries the highly infectious coronavirus,

Xiangyun Li and Kang Li are with West China Biomedical Big Data Center, West China Hospital, Sichuan University, Chengdu, Sichuan, 610041, P.R.China. (e-mail: xiangyun.app@gmail.com; likang@wchscu.cn).

Qi Lu (corresponding author) is with Department of Mechanical Engineering, Sichuan University-Pittsburgh Institute, Sichuan University, Chengdu, Sichuan, 610207, P.R.China / Nursing Key Laboratory of Sichuan Province, Sichuan University, Chengdu, Sichuan, 610207, P.R.China / Evidence-based Nursing Center, West China Hospital, Sichuan University, Chengdu, Sichuan, 610041, P.R.China. (e-mail: qi.lu@scu.edu.cn).

Jiali Chen is with Department of Orthopedics, West China Hospital, Sichuan University, Chengdu, Sichuan, 610041, P.R.China / West China School of Nursing, Sichuan University, Chengdu, Sichuan, 610041, P.R.China. (e-mail: cj185614115@163.com). putting themselves and possibly their families at high risk of infection [3]. With the benefits of using the robotic systems for combating the COVID-19, including intrinsic immunity to the virus, intensive and extra long hour service capability, process automation and multi-functional testing, the personal exposure to the patients and the labor intensity of the healthcare workers are largely reduced [4], [5]. Therefore, various researchers have investigated the use of the robot manipulators for carrying out the healthcare tasks, like throat swabbing [6], nasal swapping [7] and ultrasound testing [8].

Robot manipulator control is a inherently challenging task due to the nonlinear system dynamics, kinematic uncertainties from interchangeable end-effectors with different dimensions and dynamic uncertainties from payloads with unknown mass [9]-[11]. The static and kinematic frictions also create nonlinear disturbance causing the motor torque loss up to $30 \%$ [12]. The dynamic behavior of the manipulator is highly associated with the control system design. To deal with the aforementioned problems, the adaptive and robust controllers are usually developed to maintain performance in terms of stability, tracking error, or other specifications, despite parametric uncertainties, external disturbances and unmodeled dynamics presented in the real manipulator system.

The investigation of the adaptive control methods for robot manipulator has a long history. The employment of adaptive control provides the system with the capability of real time parametric uncertainty estimations, thus enabling the robot manipulators to perform tasks in the unknown environment [13]. In [14], Cheah et al. proposed an adaptive feedback control law for robot manipulator set-point control in task space to deal with the kinematic uncertainty. The developed controller can handle the uncertain Jacobian matrix and uncertain gravitational force with simulation validation. Then the proposed method was extended to handle the kinematic and dynamic uncertainties simultaneously in [15]. Besides the transpose Jacobian approach, the inverse Jacobian regulator has been investigated in [16]. Considering the physical constraints of the actuators, the torque amplitude limited adaptive control was proposed in [9]. To deal with the implementation issue with the industrial robot and ensure the transient performance of the system, the adaptive controllers which enjoy the separation properties were proposed in [17]. The dynamic behavior of the inner loop controller, which comes with the robot manipulator, is incorporated into the outer loop controller design, which is specified by the user, to guarantee the stability and control 
performance of the overall closed-loop system. Though the adaptive controller has the benefits of dynamic compensation, the controller development usually relies on the assumption of linearly parameterized system [18].

On the other hand, to handle the uncertain nonlinearities and external disturbance, the robust control method is usually adopted. The novel robust model predictive control that unifies the planning and control layers was proposed in [19], where the neural networks is utilized to approximate the piece-wise constant model predictive control input so that the computation time is reduced. The sliding mode control (SMC) is a nonlinear robust control method that alters the dynamics of a system by the application of a discontinuous control signal which forces the system to slide along the sliding surface. By adding adaptive term to a robust control algorithm, i.e. sliding mode controller, the superior performance of the proposed controller was demonstrated with an industrial robot manipulator in [18] with comparison to the controller without adaptive term. In [20], the integral suboptimal second order sliding mode control algorithm was developed to reduce the reaching phase and enhance the robustness; experimental results demonstrated the better robustness of the proposed method compared to conventional PD control. The time-delay control (TDC) utilizes the time-delayed information at the previous sampling constant to estimate the external disturbance. To enhance the performance of the TDC, Jin et al. adopted a nonlinear sliding modebased time delay estimation error correction term, rendering an inclusive controller structure [12]. The TDC with the adaptive gain dynamics was investigated in [21] to handle the significant payload changes for robot manipulator. Instead of using a delay term, the uncertainty and disturbance estimator (UDE)-based control [22] employs a filter to estimate the uncertainty and disturbance, thus bringing the benefits of no delay in the system and no oscillations in the control signal. The UDE-based control has been successfully applied to the motion control of various mechanical systems, including robot manipulator [23], [24], quadrotor [25] and piezoelectric stage [26]. However, in [23], [24], only the simulation validation with two-DOF planar robot is considered. Moreover, the physical human-robot interaction is not discussed.

When humans are involved, one of the essential capability for practical robot manipulator deployment is to guarantee the interaction safety [4]. Roughly speaking, there are two types of physical human-robot interactions, which are unexpected collision and intentional interaction, such as hand-shaking and cooperated manipulation. In both scenarios, the aforementioned conventional adaptive and robust motion control methods with the general aim of increasing the rigidity of the robot is not suitable for safe interaction [27]; besides the model uncertainties, nonlinearities and external disturbances, the safety and compliance requirement in physical human-robot interaction makes the robot manipulator controller development a quite challenging while fascinating research topic [4], [28]. Usually, the end-effector path in the task space is specified as a series of set-points [9], [21], [29]. For applications which involves human-robot interaction or cooperation, it is more desirable to set the target for the robot end-effector as a region instead of a point in Cartesian space. For example, a recently developed assistive feeding robot can help the handicapped patient to complete the eating task by delivering the soup using a spoon to a target area near the patient's mouth. Then the patient will guide the motion of the end-effector with the palm to accomplish final steps [30]. Another example would be the robot-assisted rehabilitation for paralyzed patient. Besides the passive training, where the patient's limb is guided by the robot to follow strict patterns repeatedly, the active participation is encouraged once the patient's limb regains even partial motor function. As for the active participation, the set-point or trajectory control may intervene the patient intended motion, making the rehabilitation process ineffective. With the control objective of region reaching for the robot, the patient can take initiative to guide the robot within a feasible region while the robot guide the patient if his limb lies outside this region, providing more flexibility for the active participation for the patient [31], [32]. In previous examples, the shared autonomy is achieved with the integration of the human inputs along with the robot automation to accommodate the uncertainty and variability in complex tasks on an as-needed basis [33], [34]. The concept of region reaching control for robot manipulator is originally proposed in [35]. Compared to the conventional set-point control, the region reaching control can provide more flexibility and it has the advantages of faster convergence with less control effort. Furthermore, the robot manipulator will exhibit compliant behavior inside the target region to ensure the safe and smooth physical humanrobot interaction. Therefore, the region reaching control has been widely investigated in robot manipulator adaptive control [36], [37], flexible-joint manipulator control [30], humanguided robotic co-manipulation [38]. However, previous works usually adopt the adaptive control method to compensate the parametric uncertainties without considering the effect of functional nonlinearities and external disturbance.

Based on the above discussions, an UDE-based robust region tracking controller for the robot manipulator is developed to achieve the region trajectory tracking and physical humanrobot interaction simultaneously. The model uncertainty and external disturbance are estimated and compensated by the UDE to ensure the accurate region trajectory tracking. The compliant behavior of the robot manipulator is achieved with the regional feedback. The seamless integration of both components are achieved by utilizing the back-stepping approach and the Lyapunov-based control design method. The main contributions of this article are listed as follows:

(1) The UDE-based controller with regional feedback is developed to simultaneously achieve the robust region trajectory tracking in the presence of model uncertainties and external disturbances and compliant physical human-robot interaction. The backstepping technique is employed to integrate the regional error feedback into the UDE-based control framework. Compared with previous UDE-based robot manipulator control works [23], [24], where only the simulation validation with two-DOF planar robot is considered, this work considers the three dimensional trajectory tracking and compliant physical human-robot interaction without force sensor using a multiDOF spatial robot manipulator with experimental validation. The experiments considered in this work is more challenging 
and realistic, making the proposed method more promising for direct application in industrial or medical scenarios.

(2) The regional feedback error is adopted for fast convergence with less control effort compared with set point control. Furthermore, the safe and smooth physical human-robot interaction is achieved within the target region. Compared with the existing works for region reaching robot manipulator control [36]-[38], the proposed method incorporate the UDE to estimate and compensate the model uncertainties and external disturbances, ensuring the reference tracking performance in the presence of unknown payload or frictions. The experimental comparison with the control method proposed in [35] is conducted to demonstrate the superior performance of the proposed method.

(3) The rigorous mathematical proof of the closed-loop system stability with the proposed UDE-based region tracking controller is provided to show the uniform boundedness of the regional tracking error in the presence of the model uncertainties and disturbances. Extensive experimental validations with a multi-DOF collaborative robot manipulator, universal robots (UR) 10, are carried out to demonstrate the effectiveness of the proposed methods, including trajectory tracking, comparison with the existing controller in the presence of model uncertainties and patient temperature measurement with compliant human-robot interaction.

The rest of this article is organized as follows. Section II presents the preliminaries about the robot manipulator dynamics and the problem formulation. Section III discusses the developments of the UDE-based robust region tracking controller design and the closed-loop system stability analysis. The experimental setup and experimental results are discussed in Section IV. Section V concludes this article.

\section{System Dynamics And PROBlem Formulation}

\section{A. System Dynamics of Robot Manipulator}

Let $\mathbf{x} \in \mathbb{R}^{3}$ represent the position vector of the end-effector in task space

$$
\mathbf{x}=H(\mathbf{q})
$$

where $\mathbf{q} \in \mathbb{R}^{n}$ is a vector of generalized joint coordinates for $n$ DOFs manipulator, $H(\mathbf{q})$ is the transformation matrix which maps the joint angle $\mathbf{q}$ from the joint space to end-effector position $\mathbf{x}$ in the task space. Taking the time derivative of (1), the kinematic relationship between the velocity vector of the end-effector $\dot{\mathbf{x}}$ and the joint velocity vector $\dot{\mathbf{q}}$ can be described as

$$
\dot{\mathbf{x}}=\mathbf{J}(\mathbf{q}) \dot{\mathbf{q}}
$$

where $\mathbf{J}(\mathbf{q}) \in \mathbb{R}^{3 \times n}$ is the Jacobian matrix. The dynamics of the robot manipulator in the joint space can be described as

$$
\mathbf{M}(\mathbf{q}) \ddot{\mathbf{q}}+\mathbf{C}(\mathbf{q}, \dot{\mathbf{q}}) \dot{\mathbf{q}}+\mathbf{g}(\mathbf{q})=\boldsymbol{\tau}+\boldsymbol{\tau}_{d}
$$

where $\mathbf{M}(\mathbf{q}) \in \mathbb{R}^{n \times n}$ is the inertia matrix, $\mathbf{g}(\mathbf{q}) \in \mathbb{R}^{n}$ denotes a gravitational torque vector, $\mathbf{C}(\mathbf{q}, \dot{\mathbf{q}}) \dot{\mathbf{q}} \in \mathbb{R}^{n}$ is the Coriolis and centrifugal torque, $\tau$ denotes the joint control torque vector and $\tau_{d}$ is the bounded disturbance vector, which is caused by the unmodeled friction and the unmodeled coupling effects due to the motion of links. From (2), $\dot{\mathbf{q}}$ and $\ddot{\mathbf{q}}$ can be derived as

$$
\begin{aligned}
& \dot{\mathbf{q}}=\mathbf{J}^{-1}(\mathbf{q}) \dot{\mathbf{x}} \\
& \ddot{\mathbf{q}}=\dot{\mathbf{J}}^{-1}(\mathbf{q}) \dot{\mathbf{x}}+\mathbf{J}^{-1}(\mathbf{q}) \ddot{\mathbf{x}}
\end{aligned}
$$

For non-redundant articulated manipulators, the task space dynamics can be derived by combining the joint space dynamics (3) and (4) [39]

$$
\mathbf{M}_{\mathbf{x}}(\mathbf{q}) \ddot{\mathbf{x}}+\mathbf{C}_{\mathbf{x}}(\mathbf{q}, \dot{\mathbf{q}}) \dot{\mathbf{x}}+\mathbf{g}_{\mathbf{x}}(\mathbf{q})=\mathbf{F}+\mathbf{J}^{-\mathbf{T}}(\mathbf{q}) \boldsymbol{\tau}_{d}(5)
$$

where

$$
\begin{aligned}
\mathbf{M}_{\mathbf{x}}(\mathbf{q}) & =\mathbf{J}^{-\mathbf{T}}(\mathbf{q}) \mathbf{M}(\mathbf{q}) \mathbf{J}^{-1}(\mathbf{q}) \\
\mathbf{C}_{\mathbf{x}}(\mathbf{q}, \dot{\mathbf{q}}) & =\mathbf{J}^{-\boldsymbol{\top}}\left(\mathbf{M}(\mathbf{q}) \dot{\mathbf{J}}^{-1}(\mathbf{q})+\mathbf{C}(\mathbf{q}, \dot{\mathbf{q}}) \mathbf{J}^{-1}(\mathbf{q})\right) \\
\mathbf{g}_{\mathbf{x}}(\mathbf{q}) & =\mathbf{J}^{-\boldsymbol{\top}} \mathbf{g}(\mathbf{q}) \\
\mathbf{F} & =\mathbf{J}^{-\boldsymbol{\top}} \boldsymbol{\tau}
\end{aligned}
$$

For the experimental validation platform used in this work, UR 10 robot, it consists of a spherical wrists, whose three joint axes intersect at a common point. The Euclidean position of the end-effector is determined by the first three joints. Therefore, the Jacobian matrix is a $3 \times 3$ square matrix. As for the redundant manipulator, since $\mathbf{J}$ is not a square matrix, the Jacobian inverse should be replaced with Moore-Penrose pseudo inverse which is defined as $\mathbf{J}^{+}=\left(\mathbf{J}^{\top} \mathbf{J}\right)^{-1} \mathbf{J}^{\top}$. In the present study, it is assumed that the robot manipulator position lies in finite workspace such that the Jacobian matrix is nonsingular and the following property holds.

Property 1: The matrix $\dot{\mathbf{M}}_{\mathbf{x}}(\mathbf{q})-2 \mathbf{C}_{\mathbf{x}}(\mathbf{q}, \dot{\mathbf{q}})$ is a skewsymmetric matrix such that $\boldsymbol{\epsilon}^{\boldsymbol{\top}}\left[\dot{\mathbf{M}}_{\mathbf{x}}(\mathbf{q})-2 \mathbf{C}_{\mathbf{x}}(\mathbf{q}, \dot{\mathbf{q}})\right] \boldsymbol{\epsilon}=\mathbf{0}$, where $\epsilon \in \mathbb{R}^{n}$ is an arbitrary column vector.

It can be observed from (5) that the robot dynamics is highly nonlinear, where the system parameter matrices $\mathbf{M}_{\mathbf{x}}(\mathbf{q}), \mathbf{C}_{\mathbf{x}}(\mathbf{q}, \dot{\mathbf{q}})$ and $\mathbf{g}_{\mathbf{x}}(\mathbf{q})$ are all functions of the joint variables. Moreover, the uncertainty that comes from the inaccurate modeling of the robot manipulator complex dynamics, the difference between the model and experimental platform parameters, and the variation of system parameters during operation, such as picking up objects, all cause problems for the controller design and accurate reference tracking. To alleviate the need of accurate robot dynamics modeling, the manipulator model is reformulated as [12]

$$
\hat{\mathbf{M}}_{\mathbf{x}}(\mathbf{q}) \ddot{\mathbf{x}}+\hat{\mathbf{C}}_{\mathbf{x}}(\mathbf{q}, \dot{\mathbf{q}}) \dot{\mathbf{x}}+\hat{\mathbf{g}}_{\mathbf{x}}(\mathbf{q})+\mathbf{D}_{\mathbf{x}}=\mathbf{F}
$$

where $\hat{\mathbf{M}}_{\mathbf{x}}(\mathbf{q}), \hat{\mathbf{C}}_{\mathbf{x}}(\mathbf{q}, \dot{\mathbf{q}})$ and $\hat{\mathbf{g}}_{\mathbf{x}}(\mathbf{q})$ are the known nominal system parameter matrix and

$$
\begin{aligned}
\mathbf{D}_{\mathbf{x}} & =\left[\mathbf{M}_{\mathbf{x}}-\hat{\mathbf{M}}_{\mathbf{x}}\right] \ddot{\mathbf{x}}+\left[\mathbf{C}_{\mathbf{x}}-\hat{\mathbf{C}}_{\mathbf{x}}\right] \dot{\mathbf{x}}+\left[\mathbf{g}_{\mathbf{x}}-\hat{\mathbf{g}}_{\mathbf{x}}\right]-\mathbf{J}^{-\boldsymbol{T}} \boldsymbol{\tau}_{d} \\
& =\tilde{\mathbf{M}}_{\mathbf{x}} \ddot{\mathbf{x}}+\tilde{\mathbf{C}}_{\mathbf{x}} \dot{\mathbf{x}}+\tilde{\mathbf{g}}_{\mathbf{x}}-\mathbf{J}^{-\boldsymbol{T}} \boldsymbol{\tau}_{d}
\end{aligned}
$$

represents lumped uncertainty term, which includes the model uncertainties $\tilde{\mathbf{M}}_{\mathbf{x}}, \tilde{\mathbf{C}}_{\mathbf{x}}, \tilde{\mathbf{g}}_{\mathbf{x}}$ and the unknown external disturbance torque $\tau_{d}$. 


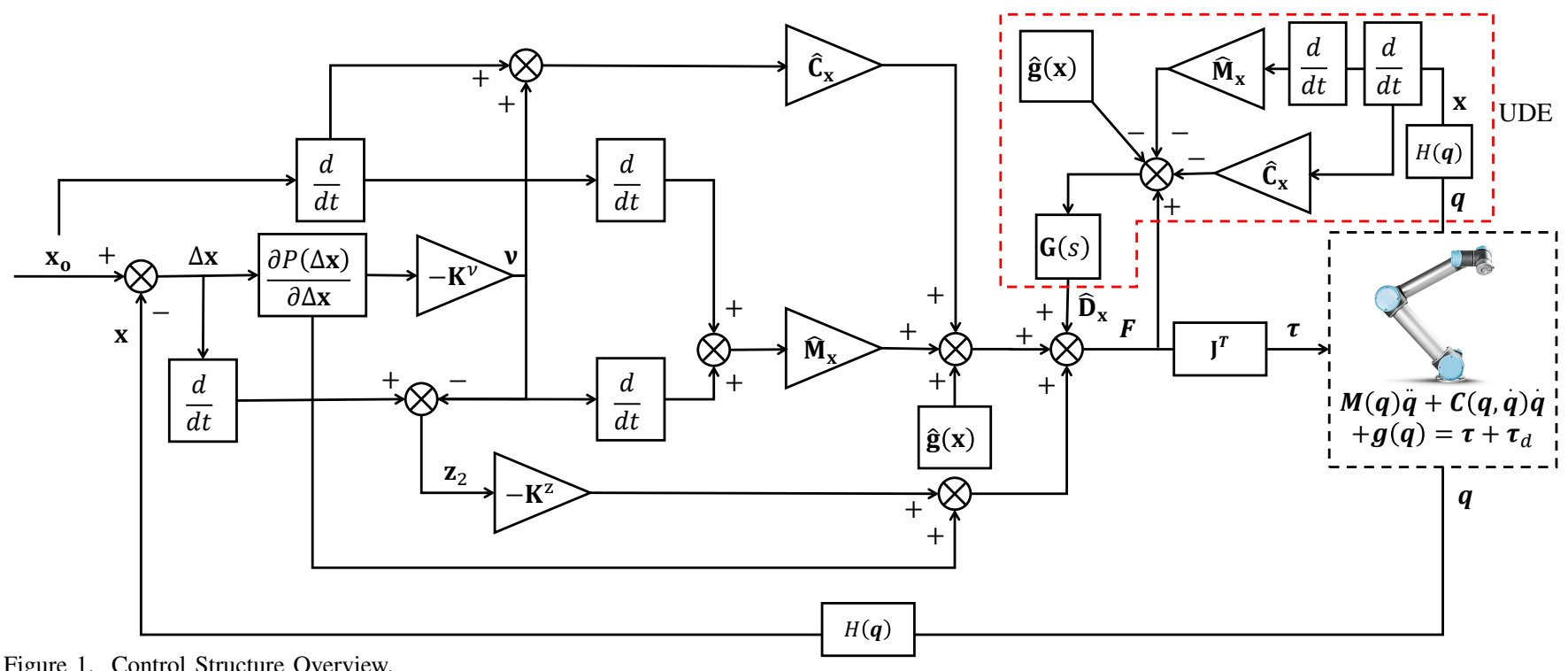

\section{B. Problem Formulation}

The control objective of this paper is two-fold: i) to design the UDE-based tracking control algorithm with regional feedback for the uncertain robot manipulator (7) such that the position of the end-effector will be regulated to track the reference target region in the task space; ii) the robot exhibits compliant behavior within the target region such that the safe human-robot interaction is achieved without utilizing the external force sensor. The desired position center for the end-effector is defined as $\mathbf{x}_{o} \in \mathbb{R}^{3}$ and its trajectory is assumed to be bounded up to its second order derivative.

\section{Controller Design And Stability AnAlysis}

\section{A. Regional Error Feedback}

The artificial potential function-based approach has been widely investigated for robotic application including robot manipulator control [40], path planning [41], and multi-agent formation control [42] with the attractive features of mathematical elegance and simplicity. Inspired by [35], to ensure the compliant human-robot interaction, the reference for the endeffector of the robot manipulator is defined as a region instead of a point. The regional feedback error is constructed as the gradient of artificial potential function. The target region can be expressed as a compact set [35], [43]

$$
\Omega=\left\{\Delta \mathbf{x} \mid f(\Delta \mathbf{x})=\|\Delta \mathbf{x}\|^{2}-r_{o}^{2} \leq 0\right\}
$$

where $\Delta \mathbf{x}=\mathbf{x}-\mathbf{x}_{o}, \mathbf{x}_{o}$ is the center of the target region, $f(\Delta \mathbf{x}): \mathbb{R}^{3} \rightarrow \mathbb{R}$ is the objective function. The target function is chosen to be continuous and differentiable with respect to $\Delta \mathbf{x}$ such that the boundedness of $f(\Delta \mathbf{x})$ assures the boundedness of its first and second partial derivatives, which are $\frac{\partial f(\cdot)}{\partial \Delta \mathbf{x}}$ and $\frac{\partial^{2} f(\cdot)}{\partial^{2} \Delta \mathbf{x}}$. The potential energy function for the manipulator is designed as

$$
P(\Delta \mathbf{x})=\frac{c}{2} \max (0, f(\Delta \mathbf{x}))^{2}
$$

where $c$ is a positive constant. The target potential energy function $P(\Delta \mathbf{x})$ is continuous differentiable with respect to $\Delta \mathrm{x}$ and monotonically increases with $\|\Delta \mathrm{x}\|$. Partially differentiating the potential function $P(\Delta \mathbf{x})$ with respect to $\Delta \mathrm{x}$ leads to

$$
\begin{aligned}
\frac{\partial P(\Delta \mathbf{x})}{\partial \Delta \mathbf{x}} & =c \max (0, f(\Delta \mathbf{x})) \frac{\partial f(\Delta \mathbf{x})}{\partial \Delta \mathbf{x}} \\
& =2 c \max (0, f(\Delta \mathbf{x})) \Delta \mathbf{x}
\end{aligned}
$$

where $\frac{\partial P(\Delta \mathbf{x})}{\partial \Delta \mathbf{x}}$ is a column vector. It can be seen that from (9) that when the manipulator end-effector is outside the target region $\Omega, \frac{\partial P(\Delta \mathbf{x})}{\partial \Delta \mathbf{x}}$ is activated to drive the manipulator endeffector towards the target region and when the manipulator end-effector is inside the target region $\Omega, \frac{\partial P(\Delta \mathbf{x})}{\partial \Delta \mathbf{x}}$ remains as zero.

\section{B. UDE-based Controller Design}

In this subsection, the UDE-based task space controller with regional feedback for the robot manipulator is designed with the integrator backstepping technique. The overview of the controller structure is shown in Fig. 1. To facilitate the controller design, the following error coordinate transformation is utilized

$$
\begin{aligned}
& \mathbf{z}_{1}=P(\Delta \mathbf{x}) \\
& \mathbf{z}_{2}=\Delta \dot{\mathbf{x}}-\boldsymbol{\nu}
\end{aligned}
$$

where $\boldsymbol{\nu}$ is the virtual controller to be designed. Taking the time derivative of (11) leads to

$$
\begin{aligned}
\dot{\mathbf{z}}_{1} & =\left[\frac{\partial P(\Delta \mathbf{x})}{\partial \Delta \mathbf{x}}\right]^{\top} \Delta \dot{\mathbf{x}} \\
& =\left[\frac{\partial P(\Delta \mathbf{x})}{\partial \Delta \mathbf{x}}\right]^{\top}\left(\mathbf{z}_{2}+\boldsymbol{\nu}\right) \\
& =\left[\frac{\partial P(\Delta \mathbf{x})}{\partial \Delta \mathbf{x}}\right]^{\top} \mathbf{z}_{2}-\left[\frac{\partial P(\Delta \mathbf{x})}{\partial \Delta \mathbf{x}}\right]^{\top} \mathbf{K}^{\nu} \frac{\partial P(\Delta \mathbf{x})}{\partial \Delta \mathbf{x}}
\end{aligned}
$$

where the virtual controller $\nu$ is designed in the form of

$$
\boldsymbol{\nu}=-\mathbf{K}^{\nu} \frac{\partial P(\Delta \mathbf{x})}{\partial \Delta \mathbf{x}}
$$


and $\mathbf{K}^{\nu}$ is a positive constant symmetric matrix. Consider the following controller

$$
\begin{aligned}
\mathbf{F} & =\hat{\mathbf{M}}_{\mathbf{x}}\left(\ddot{\mathbf{x}}_{o}+\dot{\nu}\right)+\hat{\mathbf{C}}_{\mathbf{x}}\left(\dot{\mathbf{x}}_{o}+\nu\right)+\hat{\mathbf{g}}_{\mathbf{x}}+\hat{\mathbf{D}}_{\mathbf{x}} \\
& -\mathbf{K}^{z} \mathbf{z}_{2}-\frac{\partial P(\Delta \mathbf{x})}{\partial \Delta \mathbf{x}}
\end{aligned}
$$

where $\hat{\mathbf{D}}_{\mathbf{x}}$ is the lumped uncertainty estimation term which will be designed using the UDE technique and $\mathbf{K}^{z}$ is a positive constant symmetric matrix. By combing (15) and (7), then the closed loop system dynamics become

$$
\hat{\mathbf{M}}_{\mathbf{x}} \dot{\mathbf{z}}_{2}+\hat{\mathbf{C}}_{\mathbf{x}} \mathbf{z}_{2}-\tilde{\mathbf{D}}_{\mathbf{x}}+\frac{\partial P(\Delta \mathbf{x})}{\partial \Delta \mathbf{x}}+\mathbf{K}^{z} \mathbf{z}_{2}=\mathbf{0}
$$

where $\tilde{\mathbf{D}}_{\mathbf{x}}=\hat{\mathbf{D}}_{\mathbf{x}}-\mathbf{D}_{\mathbf{x}}$ is the lumped uncertainty estimation error. Next, the lumped uncertainty term estimation will be constructed. From the system dynamics (7), it can solved that

$$
\mathbf{D}_{\mathbf{x}}=\mathbf{F}-\hat{\mathbf{M}}_{\mathbf{x}} \ddot{\mathbf{x}}-\hat{\mathbf{C}}_{\mathbf{x}} \dot{\mathbf{x}}-\hat{\mathbf{g}}_{\mathbf{x}}
$$

By adopting stable strictly proper filters with unity steady-state gains along with (12), the UDE technique provided in [22] is used to construct the lumped uncertainty term estimation

$$
\begin{aligned}
\hat{\mathbf{D}}_{\mathbf{x}}= & \mathcal{L}^{-1}\{\mathbf{G}(s)\} *\left[\mathbf{F}-\hat{\mathbf{M}}_{\mathbf{x}} \ddot{\mathbf{x}}-\hat{\mathbf{C}}_{\mathbf{x}} \dot{\mathbf{x}}-\hat{\mathbf{g}}_{\mathbf{x}}\right] \\
= & \mathcal{L}^{-1}\{\mathbf{G}(s)\} *\left[\mathbf{F}-\hat{\mathbf{M}}_{\mathbf{x}}\left(\ddot{\mathbf{x}}_{o}+\dot{\boldsymbol{\nu}}+\dot{\mathbf{z}}_{2}\right)\right. \\
& \left.-\hat{\mathbf{C}}_{\mathbf{x}}\left(\dot{\mathbf{x}}_{o}+\boldsymbol{\nu}+\mathbf{z}_{2}\right)-\hat{\mathbf{g}}_{\mathbf{x}}\right]
\end{aligned}
$$

Substituting (17) back into (15) and solving for the control inputs $\mathbf{F}$ results in

$$
\begin{aligned}
\mathbf{F} & =\hat{\mathbf{M}}_{\mathbf{x}}\left(\ddot{\mathbf{x}}_{o}+\dot{\nu}\right)+\hat{\mathbf{C}}_{\mathbf{x}}\left(\dot{\mathbf{x}}_{o}+\nu\right)+\hat{\mathbf{g}}_{\mathbf{x}} \\
& -\mathcal{L}^{-1}\left\{\left(\mathbb{I}_{3}-\mathbf{G}(s)\right)^{-1}\right\} *\left(\mathbf{K}^{z} \mathbf{z}_{2}+\frac{\partial P(\Delta \mathbf{x})}{\partial \Delta \mathbf{x}}\right) \\
& -\mathcal{L}^{-1}\left\{\left(\mathbb{I}_{3}-\mathbf{G}(s)\right)^{-1} \mathbf{G}(s)\right\} *\left(\hat{\mathbf{M}}_{\mathbf{x}} \dot{\mathbf{z}}_{2}+\hat{\mathbf{C}}_{\mathbf{x}} \mathbf{z}_{2}\right)
\end{aligned}
$$

where $\mathbb{I}_{n} \in \mathbb{R}^{n \times n}$ is the $n$-dimensional identity matrix. To map the task space control inputs into the joint torque, from (6), the control torques can be calculated as

$$
\boldsymbol{\tau}=\mathbf{J}^{\top} \mathbf{F}
$$

\section{Stability Analysis}

Consider the following Lyapunov-like function candidate

$$
V=\frac{1}{2} \mathbf{z}_{2}^{\top} \hat{\mathbf{M}}_{\mathbf{x}} \mathbf{z}_{2}+\mathbf{z}_{1}
$$

Take the time derivative of (20) leads to

$$
\dot{V}=\mathbf{z}_{2}^{\top} \hat{\mathbf{M}}_{\mathbf{x}} \dot{\mathbf{z}}_{2}+\frac{1}{2} \mathbf{z}_{2}^{\top} \dot{\hat{\mathbf{M}}}_{\mathbf{x}} \mathbf{z}_{2}+\dot{\mathbf{z}}_{1}
$$

Substituting (13) and (16) into (21) leads to

$$
\begin{aligned}
& \dot{V}=\mathbf{z}_{2}^{\top}\left(\hat{\mathbf{M}}_{\mathbf{x}} \dot{\mathbf{z}}_{2}\right)+\frac{1}{2} \mathbf{z}_{2}^{\top} \dot{\hat{\mathbf{M}}}_{\mathbf{x}} \mathbf{z}_{2}+\dot{\mathbf{z}}_{1} \\
& =\mathbf{z}_{2}^{\boldsymbol{\top}}\left[-\hat{\mathbf{C}}_{\mathbf{x}} \mathbf{z}_{2}-\left[\frac{\partial P(\Delta \mathbf{x})}{\partial \Delta \mathbf{x}}\right]-\mathbf{K}^{z} \mathbf{z}_{2}+\tilde{\mathbf{D}}_{\mathbf{x}}\right] \\
& +\frac{1}{2} \mathbf{z}_{2}^{\top} \dot{\hat{\mathbf{M}}}_{\mathbf{x}} \mathbf{z}_{2}+\left[\frac{\partial P(\Delta \mathbf{x})}{\partial \Delta \mathbf{x}}\right]^{\top} \mathbf{z}_{2}-\left[\frac{\partial P(\Delta \mathbf{x})}{\partial \Delta \mathbf{x}}\right]^{\top} \mathbf{K}^{\nu} \frac{\partial P(\Delta \mathbf{x}}{\partial \Delta \mathbf{x}} \\
& =-\mathbf{z}_{2}^{\top} \mathbf{K}^{z} \mathbf{z}_{2}+\frac{1}{2} \mathbf{z}_{2}^{\top}\left[\dot{\hat{\mathbf{M}}}_{\mathbf{x}}-2 \hat{\mathbf{C}}_{\mathbf{x}}\right] \mathbf{z}_{2} \\
& -\left[\frac{\partial P(\Delta \mathbf{x})}{\partial \Delta \mathbf{x}}\right]^{\top} \mathbf{K}^{\nu} \frac{\partial P(\Delta \mathbf{x})}{\partial \Delta \mathbf{x}}+\mathbf{z}_{2}^{\top} \tilde{\mathbf{D}}_{\mathbf{x}}
\end{aligned}
$$

Utilizing the Property 1 , the term $\frac{1}{2} \mathbf{z}_{2}^{\mathbf{\top}}\left[\dot{\hat{\mathbf{M}}}_{\mathbf{x}}-\hat{\mathbf{C}}_{\mathbf{x}}\right] \mathbf{z}_{2}$ becomes zero. With the Cauchy-Schwarz inequality, the above equation can be simplified as

$$
\begin{aligned}
\dot{V} \leq & -\left[\frac{\partial P(\Delta \mathbf{x})}{\partial \Delta \mathbf{x}}\right]^{\top} \mathbf{K}^{\nu} \frac{\partial P(\Delta \mathbf{x})}{\partial \Delta \mathbf{x}}-\mathbf{z}_{2}^{\top} \mathbf{K}^{z} \mathbf{z}_{2}+\left\|\mathbf{z}_{2}\right\|\left\|\tilde{\mathbf{D}}_{\mathbf{x}}\right\| \\
\leq & -\left[\frac{\partial P(\Delta \mathbf{x})}{\partial \Delta \mathbf{x}}\right]^{\top} \mathbf{K}^{\nu} \frac{\partial P(\Delta \mathbf{x})}{\partial \Delta \mathbf{x}}-\mathbf{z}_{2}^{\top}(1-\Theta) \mathbf{K}^{z} \mathbf{z}_{2} \\
& -\left\|\mathbf{z}_{2}\right\|\left(\Theta \lambda_{\min }\left(\mathbf{K}^{z}\right)\left\|\mathbf{z}_{2}\right\|-\left\|\tilde{\mathbf{D}}_{\mathbf{x}}\right\|\right) \\
\leq & -\left[\frac{\partial P(\Delta \mathbf{x})}{\partial \Delta \mathbf{x}}\right]^{\top} \mathbf{K}^{\nu} \frac{\partial P(\Delta \mathbf{x})}{\partial \Delta \mathbf{x}}-\mathbf{z}_{2}^{\top}(1-\Theta) \mathbf{K}^{z} \mathbf{z}_{2} \leq 0 \\
& \forall\left\|\mathbf{z}_{2}\right\| \geq \frac{\left\|\tilde{\mathbf{D}}_{\mathbf{x}}\right\|}{\Theta \lambda_{\min }\left(\mathbf{K}^{z}\right)}
\end{aligned}
$$

where $0<\Theta<1$ is a constant and $\lambda_{\min }(\cdot)$ represents the minimum eigenvalue operator for a matrix. As $\tilde{\mathbf{D}}_{\mathbf{x}}=$ $\hat{\mathbf{D}}_{\mathbf{x}}-\mathbf{D}_{\mathbf{x}}=\mathcal{L}^{-1}\left\{\mathbf{G}(s)-\mathbb{I}_{3}\right\} * \mathbf{D}_{\mathbf{x}}$, the derived condition $\left\|\mathbf{z}_{2}\right\| \geq \frac{\left\|\tilde{\mathbf{D}}_{\mathbf{x}}\right\|}{\Theta \lambda_{\min }\left(\mathbf{K}^{z}\right)}$ can be satisfied with the proper choice of the constant $\Theta$, the filter matrix $\mathbf{G}(s)$ and the controller parameter matrix $\mathbf{K}^{z}$. Since $V \geq 0$ and $\dot{V} \leq 0$ for all $\left\|\mathbf{z}_{2}\right\| \geq \frac{\left\|\tilde{\mathbf{D}}_{\mathbf{x}}\right\|}{\Theta \lambda_{\min }\left(\mathbf{K}^{z}\right)}$, then there exists an invariant compact set $\Omega_{V}=\{V \in \mathbb{R}: V \leq \delta\}$ such that $V$ will remain in $\Omega_{V}$ for all $t \geq 0$ with $\delta$ being the upper bound of $V$. The boundedness of $P(\Delta \mathbf{x})$ and $\mathbf{z}_{2}$ can be derived from the boundedness of $V$. The boundedness of $P(\Delta \mathbf{x})$ leads to the boundedness of $f(\Delta \mathbf{x})$ and $\Delta \mathbf{x}$. As $\mathbf{z}_{2}=\Delta \dot{\mathbf{x}}-\boldsymbol{\nu}$ is bounded, therefore, $\frac{\partial P(\Delta \mathbf{x})}{\partial \Delta \mathbf{x}}$ and $\Delta \dot{\mathbf{x}}$ are also bounded.

Consider the case where the filter matrix $\mathbf{G}(s)$ is properly designed to achieve the asymptotic estimation performance such that $\tilde{\mathbf{D}}_{\mathbf{x}}=\mathbf{0}$ as discussed in [44]. Then the time derivative of the Lyapunov-like function become

$$
\dot{V}=-\mathbf{z}_{2}^{\top} \mathbf{K}^{z} \mathbf{z}_{2}-\left[\frac{\partial P(\Delta \mathbf{x})}{\partial \Delta \mathbf{x}}\right]^{\top} \mathbf{K}^{\nu} \frac{\partial P(\Delta \mathbf{x})}{\partial \Delta \mathbf{x}} \leq 0
$$

Then $\dot{V}$ is negative everywhere and $\dot{V}=0$ only if $\mathbf{z}_{2}=0$ and $\frac{\partial P(\Delta \mathbf{x})}{\partial \Delta \mathbf{x}}=0$, which also leads to $\Delta \dot{\mathbf{x}}=0$. Since $-\mathbf{z}_{2}^{\top} \mathbf{K}^{z} \mathbf{z}_{2}-$ $\left[\frac{\partial P(\Delta \mathbf{x})}{\partial \Delta \mathbf{x}}\right]^{\top} \mathbf{K}^{\nu} \frac{\partial P(\Delta \mathbf{x})}{\partial \Delta \mathbf{x}}$ is a continuous function, according to the LaSalle Yoshizawa theorem [45]

$$
\lim _{t \rightarrow \infty} \mathbf{z}_{2}^{\top} \mathbf{K}^{z} \mathbf{z}_{2}+\left[\frac{\partial P(\Delta \mathbf{x})}{\partial \Delta \mathbf{x}}\right]^{\top} \mathbf{K}^{\nu} \frac{\partial P(\Delta \mathbf{x})}{\partial \Delta \mathbf{x}}=0
$$

Therefore, it can be concluded that $\mathbf{z}_{2} \rightarrow \mathbf{0}$ and $\frac{\partial P(\Delta \mathbf{x})}{\partial \Delta \mathbf{x}} \rightarrow \mathbf{0}$ as time tends to infinity. From (10), $\frac{\partial P(\Delta \mathbf{x})}{\partial \Delta \mathbf{x}}=\mathbf{0}$ only if $\max (0, f(\Delta \mathbf{x}))=0$ or $\Delta \mathbf{x}=\mathbf{0}$. Therefore, the robot manipulator end-effector converges to the desired region eventually as $t \rightarrow \infty$. Then, it is shown that if the filter $\mathbf{G}(s)$ is designed to achieve the asymptotic estimation performance, the proposed controller (18) will drive the end-effector to the desired region such that $\lim _{t \rightarrow \infty} \frac{\partial P(\Delta \mathbf{x})}{\partial \Delta \mathbf{x}}=\mathbf{0}$ and $\lim _{t \rightarrow \infty} \Delta \dot{\mathbf{x}}=\mathbf{0}$. If there exist non-zero estimation error, i.e. $\left\|\tilde{\mathbf{D}}_{\mathbf{x}}\right\|>0$, then $\frac{\partial P(\Delta \mathbf{x})}{\partial \Delta \mathbf{x}}$ and $\Delta \dot{\mathbf{x}}$ will be bounded. 


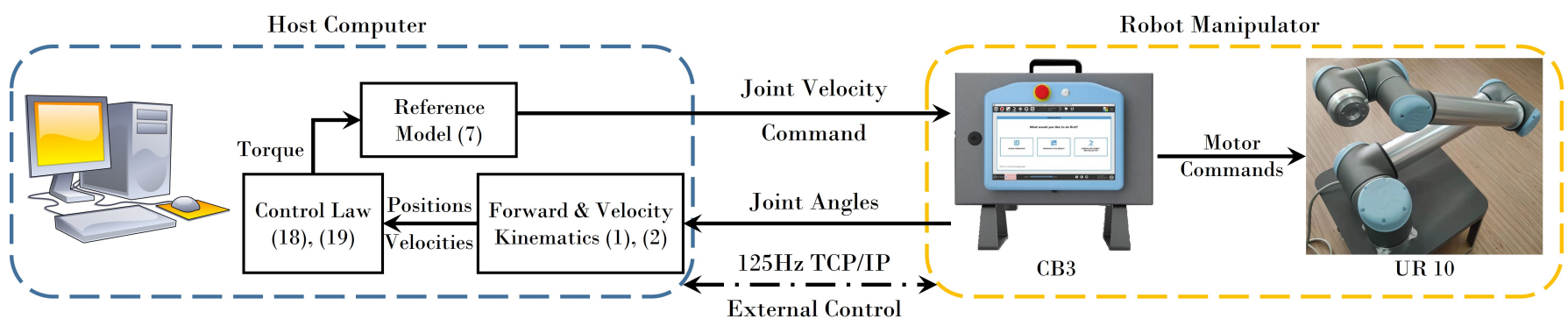

Figure 2. Experimental setup. The host computer and the robot manipulator communicates via the $125 \mathrm{~Hz}$ TCP/IP protocol. Based on the forward and velocity kinematics (1), (2), and the joint angles from the robot manipulator, the end-effector positions and velocities are determined. The control law (18), (19) calculates the joint torques, which drives the reference model. The reference model is built based on (7) without the lumped unceratinty term $\mathbf{D}_{\mathbf{x}}$. The reference model calculates the joint velocity references and send them to the CB 3 control box.

Table I

THE DH PARAMETER OF THE UR 10 ROBOT

\begin{tabular}{|c|c|c|c|}
\hline Parameter & $a(\mathrm{~m})$ & $d(\mathrm{~m})$ & $\alpha(\mathrm{rad})$ \\
\hline \hline Joint 1 & 0 & 0.118 & $\frac{\pi}{2}$ \\
\hline Joint 2 & -0.6127 & 0 & 0 \\
\hline Joint 3 & -0.5716 & 0 & 0 \\
\hline Joint 4 & 0 & 0.1639 & $\frac{\pi}{2}$ \\
\hline Joint 5 & 0 & 0.1157 & $-\frac{\pi}{2}$ \\
\hline Joint 6 & 0 & 0.0922 & 0 \\
\hline
\end{tabular}

\section{EXPERIMENTAL RESULts}

\section{A. Experimental Setup}

To validate the effectiveness of the developed UDE-based robust region tracking controller, the experimental studies are carried out with the setup shown in Fig. 2. The host computer is configured with a Core-i7 $3.00 \mathrm{Ghz}$ octa-core processor and a $32 \mathrm{~GB}$ memory. The operating system is Linux Ubuntu 18.04 and the Robot Operating System (ROS) Melodic is utilized as the software framework for robot state data acquisition, controller implementation and communication. The communication between the host computer and the robot manipulator is achieved via the TCP/IP protocol with the update rate of $125 \mathrm{~Hz}$. The joint angle data of the UR 10 robot are sent to host computer and the joint angular velocities are approximated using finite difference method. Then the forward kinematics (1) and velocity kinematics (2) are used to calculate the end-effector positions and velocities, which are then fed to the developed controller (18), (19). Due to the limitation of the experimental platform UR 10, where the joint torques cannot be directly controlled, which are quite common for industrial robot manipulators, the proposed approach is indirectly implemented by sending the joint velocity commands. The effectiveness of this method has already been validated in [17], [46]. A reference model is built based on the (7) without the lumped uncertainty term $\mathbf{D}_{\mathbf{x}}$. With the joint torques generated from the the controller (18), (19), the reference model calculates the joint velocity commands for each joint.

The UR 10 robot manipulator which has six revolute joints, a load capacity of $10 \mathrm{~kg}$ and an operation range of $1300 \mathrm{~mm}$ is used as the validation platform. The Denavit and Hartenberg (DH) parameters of the UR 10 robot manipulator are shown in Table. I. From the geometry of the robot manipulator, it can be seen that the position and orientation control of the endeffector for the UR 10 robot is decoupled. The spherical wrists are adopted, whose three joint axes intersect at a common point. Therefore, the Euclidean position of the end-effector is determined by the first three joints in the arm while the orientation depends on the motion of the wrist. In this work, since the only the position of the end-effector control is considered, the control algorithm is implemented using the first three joints while leaving the spherical wrist joints as fixed values.

Table II

Controller Parameter Selection for UR10

\begin{tabular}{|c|c|c|c|}
\hline$i$ th joint & $T_{i}$ & $k_{i}^{\nu}$ & $k_{i}^{z}$ \\
\hline \hline 1 & $1 / 30$ & 1 & 1 \\
\hline 2 & $1 / 30$ & 1 & 1 \\
\hline 3 & $1 / 30$ & 1 & 1 \\
\hline
\end{tabular}

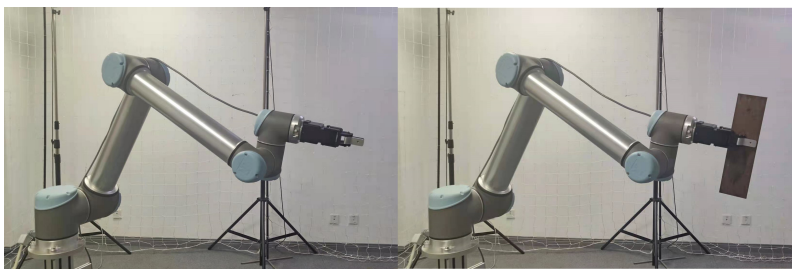

Figure 3. Case I: comparison with the existing controller. Additional payload which is unknown to the controller is added as model uncertainty.

In practice, the UDE filter is usually designed as lowpass filters for simple controller implementation [22], [44]. Therefore, the filter matrix $\mathbf{G}(s)$ is designed as $\mathbf{G}(s)=$ $\operatorname{diag}\left(\frac{1}{T_{1} s+1}, \frac{1}{T_{2} s+1}, \frac{1}{T_{3} s+1}\right)$. Similarly, the controller parameter matrices $\mathbf{K}^{\nu}, \mathbf{K}^{z}$ in (18) are designed as diagonal matrices, $\mathbf{K}^{\nu}=\operatorname{diag}\left(k_{1}^{\nu}, k_{2}^{\nu}, k_{3}^{\nu}\right)$ and $\mathbf{K}^{z}=\operatorname{diag}\left(k_{1}^{z}, k_{2}^{z}, k_{3}^{z}\right)$ with $k_{i}^{\nu}, i=1 \ldots 3$ and $k_{i}^{z}, i=1 \ldots 3$ being the positive controller constant. The positive constant $c$ is set as $c=1$. The selections of the controller parameters are listed in Table. II.

\section{B. Case I: Region Trajectory Tracking}

The comparative experiments with the region reaching controller (RRC) which is proposed in [35] are carried out to demonstrate the robustness of the proposed control strategy. The control objective of this case is to drive the end-effector of the UR10 robot to track the desired trajectory in the presence of model uncertainties. The initial positions of the 


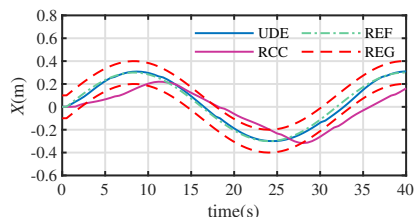

(a)

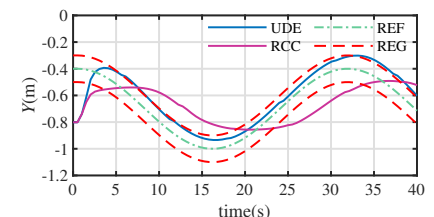

(b)

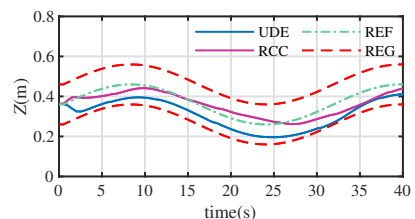

(c)

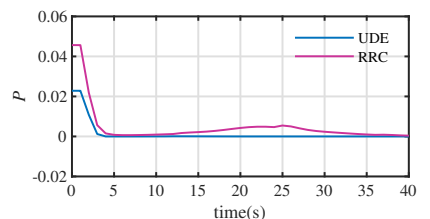

(d)

Figure 4. Case I: time series of the end-effector trajectory and potential energy function. UDE: end-effector trajectory for the proposed controller, RRC: end-effector trajectory for the region reaching controller [35], REF: reference trajectory, REG: target region. (a), (b) and (c) are the end-effector trajectory in the Cartesian space; (d) is the evolution of the potential energy function.

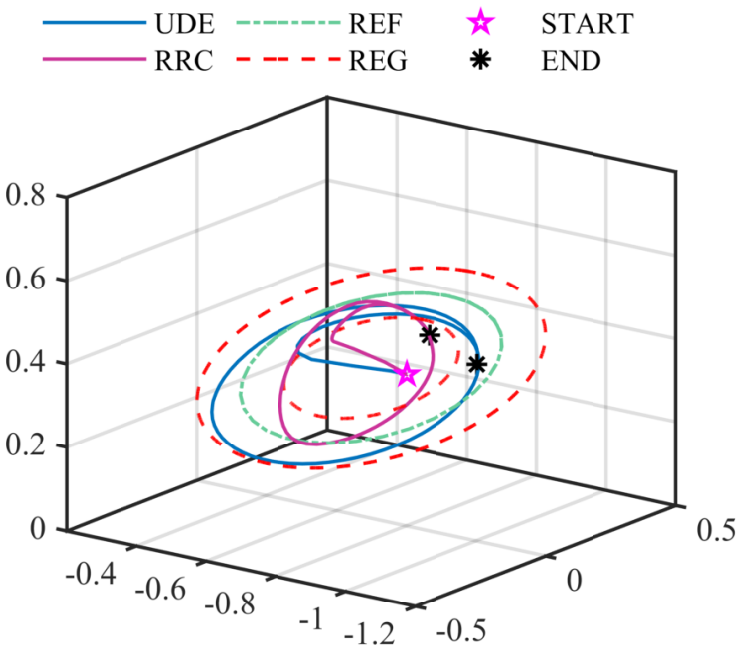

Figure 5. Case I: 3D view of the end-effector trajectory. UDE: end-effector trajectory for the proposed controller, RRC: end-effector trajectory for the region reaching controller [35], REF: reference trajectory, REG: target region, START: starting point, END: ending point.

first three joints are $\left[\begin{array}{lll}\pi / 2 & -\pi / 3 & \pi / 2\end{array}\right]^{\top}$ rad. An additional weight is added to the robot manipulator end-effector gripper at the beginning of the experiment as shown in Fig. 3. The ball shape region radius is set as $r=0.2 \mathrm{~m}$ and the three dimensional reference trajectory is set as $\mathbf{x}_{o}=$ $[0.3 * \cos (0.05 * t),-0.7+0.3 \sin (0.05 * t), 0.36$ $+0.1 \sin (t)]^{\top} \mathrm{m}$. The task space controller in [35] can be written as

$$
\boldsymbol{\tau}=-\mathbf{K}_{v} \dot{\mathbf{q}}-\mathbf{J}^{\top} k_{p} \frac{\partial P(\Delta \mathbf{x})}{\partial \Delta \mathbf{x}}+\mathbf{g}(\mathbf{q})
$$

The controller parameters are set as $\mathbf{K}_{v}=\operatorname{diag}(1,1,1)$ and $k_{p}=1$. As shown in Fig. 4, the time series of the task space end-effector trajectory, and Fig. 5, the 3D views of the task space end-effector trajectory, the RRC can not properly handle the model uncertainties which are brought by the unkonwn payload and the robot end-effector position deviates from the reference; moreover, the end-effector position can not remain within the desired region, which may potentially cause collisions with the environment. On the contrary, the UDEbased region tracking controller can successfully compensate the effects of the model uncertainties while ensuring the endeffector trajectory within the target region.

The time evolution of the potential energy function is shown in Fig. 3 (d). Fig. 6 illustrates the joints angles and
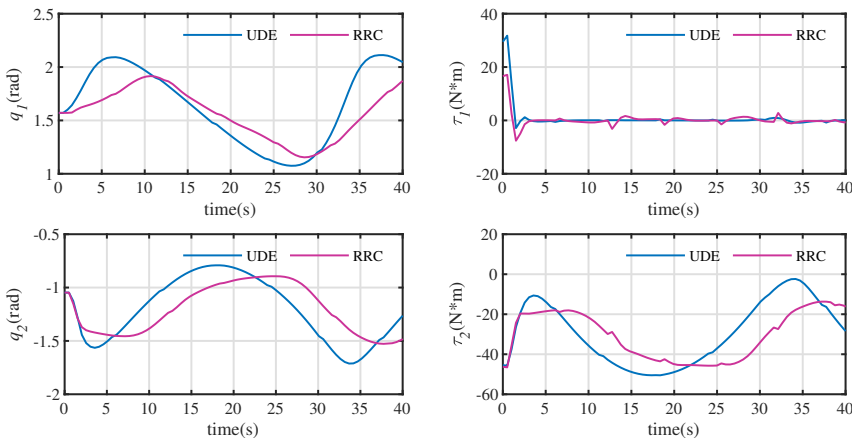

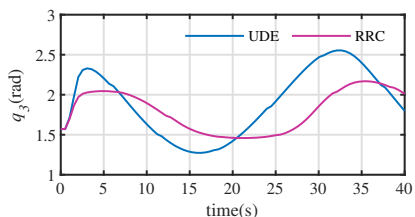

(a)

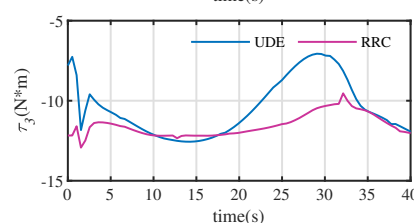

(b)
Figure 6. Case I: joint angles and control inputs. UDE: proposed controller, RRC: region reaching controller [35]. (a) joint angles (b) control inputs.

control inputs. The experimental results have demonstrated the robustness of the developed control strategies compared to the conventional region reaching controller [35] for handling model uncertainties, which is one of the essential capabilities for the robot manipulator since the end-effector tools varies for different tasks.

\section{Case II: Human-Robot Interaction}

The physical human-robot interaction capability of the proposed controller is demonstrated in this case. The control objective is to make the robot manipulator comply with the human operator within the target region, as illustrated in Fig. 7. The initial angles of the joints are set as the same as the experimental case I. The task for the robot manipulator is to take the forehead temperature measurement of the patients with a noncontact forehead thermometer installed at the end-effector, as shown in Fig. 7. The different heights of the patients may lead to uncertain target position, making the complete automation very complex or impossible. To accommodate this uncertainty resulting from this complex task, the autonomous control of the robot manipulator is blended with the human input in a shared autonomy manner [33] such that the goal is achieved with the collaboration of the robot manipulator and human operator.

The experimental procedures which are illustrated in Fig. 7, are as follows. Firstly, the robot manipulator is controlled from 

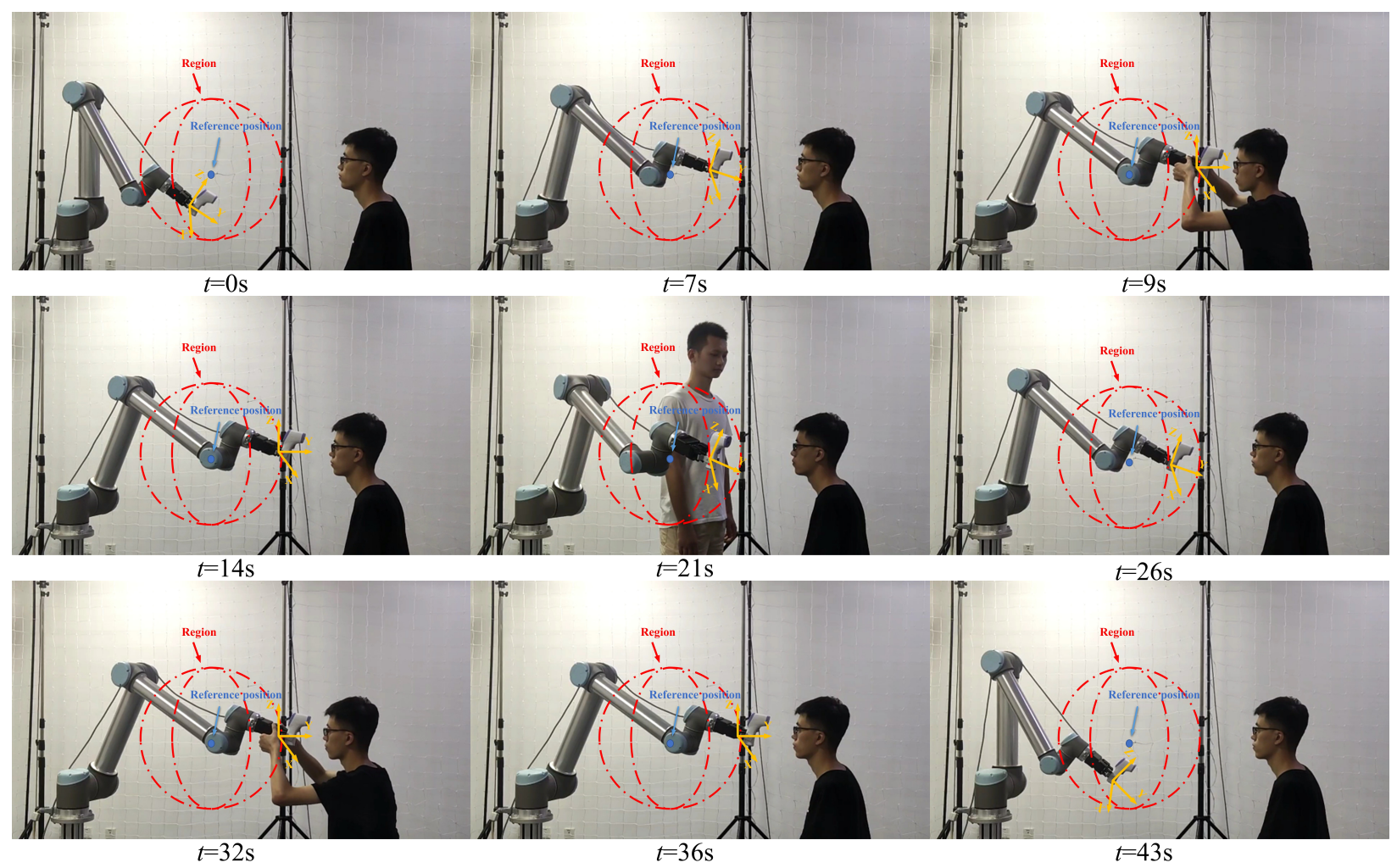

Figure 7. Case II: patient temperature measurement snapshots. $t=0-7 \mathrm{~s}$ : manipulator is controlled to reach the target region; $t=9-14 \mathrm{~s}$ : human subject adjust thermometer position to aim at his forehead; $t=21 \mathrm{~s}$ : temperature reading process is interrupted by an visitor. $t=26 \mathrm{~s}$ : robot manipulator end-effector converge back to the target region. $t=32-36 \mathrm{~s}$ : human subject re-aim thermometer and continue the temperature measurement process. $t=43 \mathrm{~s}$ : the robot manipulator retracts to its original position.

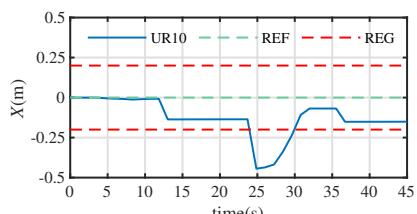

(a)

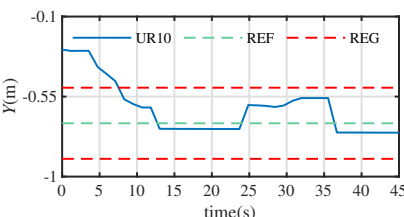

(b)

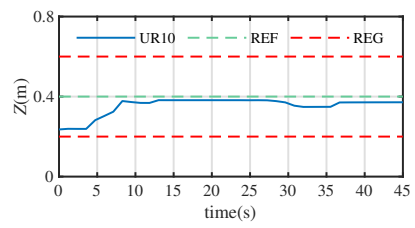

(c)

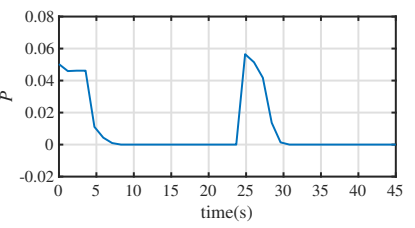

(d)

Figure 8. Case II: time series of the end-effector trajectory and potential energy function. REG: target region, UR10: robot manipulator end-effector trajectory, REF: reference trajectory. (a), (b) and (c) are the end-effector trajectory in the Cartesian coordinate directions, respectively; (d) is the evolution of the potential energy function.

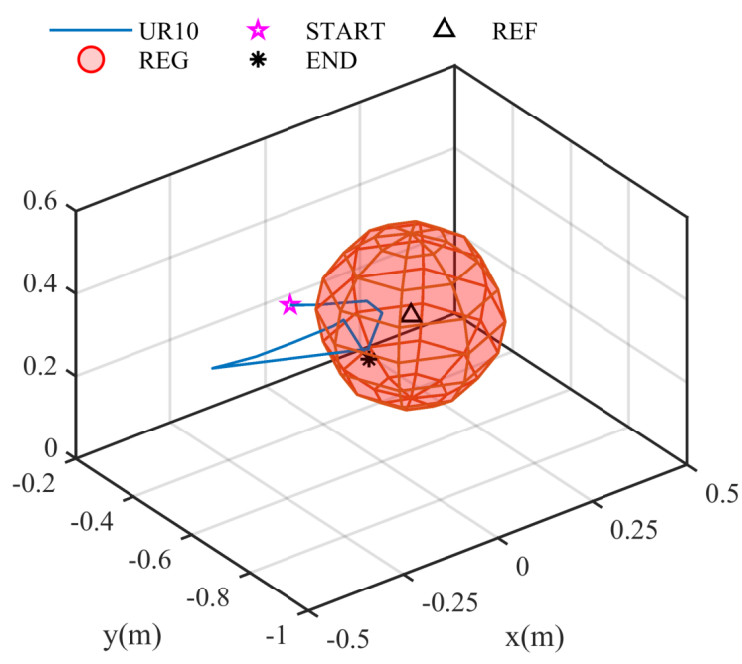

Figure 9. Case II: 3D view of the end-effectortrajectory. REG: target region, UR10: robot manipulator end-effector trajectory, START: starting point, END: ending point.

the initial position to the target region with the center being $\mathbf{x}_{o}=[0,-0.70,0.4]^{\top} \mathrm{m}$ and the radius being $r=0.2 \mathrm{~m}$.
The center of the target region is set near the forehead of the human subject such that he can reach the robot manipulator and move the thermometer to the desired position. After the robot manipulator is stabilized within the target region at around $t=7 \mathrm{~s}$, the human subject guides the thermometer to point to his forehead with his hand. From the snapshots at around $t=9 \mathrm{~s}$ and around $t=14 \mathrm{~s}$ in Figs. 7 and the endeffector trajectory shown in Figs. 8 and 9, it can be seen that the human subject can freely drag the robot manipulator to the desired position within the target region, where $f(\Delta \mathbf{x}) \leq 0$. Furthermore, with the cooperation of the human subject, the end-effector will stay at the desired position to accomplish the temperature reading task. Thirdly, an visitor enters the scene at around $t=21 \mathrm{~s}$ and he unintentionally interacts with the robot manipulator. The process of the temperature reading is interrupted by this visitor. As shown in the $t=21 \mathrm{~s}$ snapshot of the Fig. 7 and the Fig. 8 (a), the end-effector is outside the target region due to this unintentional interaction with the visitor. Then the control algorithm brings the end-effector back to the target region at $t=26 \mathrm{~s}$. Fourthly, the human subject adjusts the position of thermometer to re-aim to his forehead to continue the temperature measurement process at 


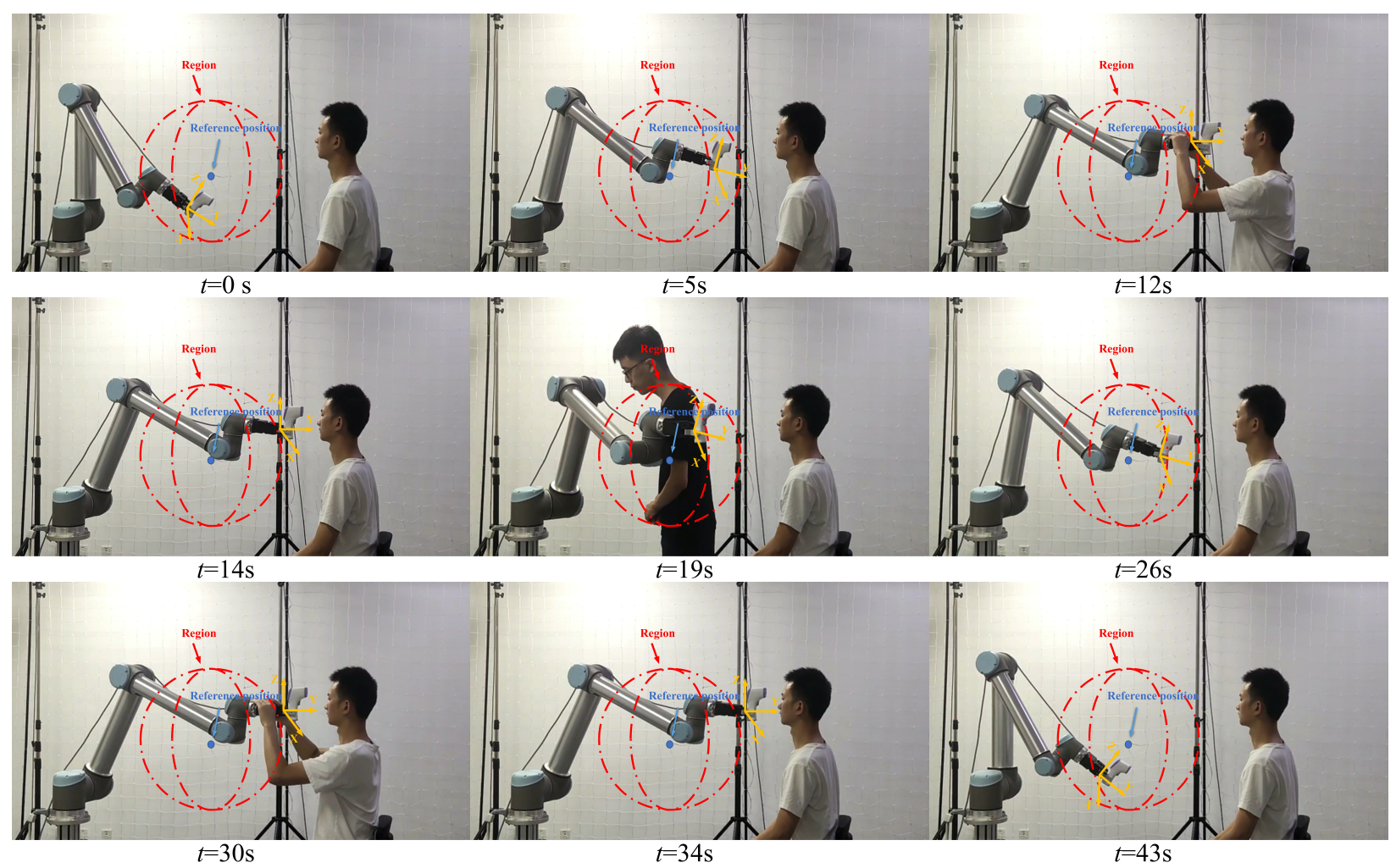

Figure 10. Case II: patient temperature measurement snapshots. $t=0-5 \mathrm{~s}$ : manipulator is controlled to reach the target region; $t=5-14 \mathrm{~s}$ : human subject adjust thermometer position to aim at his forehead; $t=19 \mathrm{~s}$ : temperature reading process is interrupted by an visitor. $t=26 \mathrm{~s}$ : robot manipulator end-effector converge back to the target region. $t=30-34 \mathrm{~s}$ : human subject re-aim thermometer and continue the temperature measurement process. $t=43 \mathrm{~s}$ : the robot manipulator retracts to its original position.

$t=32 \mathrm{~s}$. After the temperature measurement is done, the robot manipulator retracts to its original position. The time evolution of the potential energy function is shown in Fig. 8 (d) and the three dimensional views of the end-effector trajectory are shown in Fig. 9. To verify the adaptivity of the proposed approach, the same temperature measurement experiments are carried out with a different human subject, as shown in Fig 10. Based on the experimental results, it can be concluded that the proposed controller can ensure the complaint physical humanrobot interaction within the target region and stays at the point where the end-effector is released. When the robot manipulator end-effector is outside the target region, the control algorithm can bring the end-effector back to the target region. Moreover, the developed method can adapt to the variation of the human subjects for achieving the temperature measurement task.

\section{CONClusion}

To deal with the practical challenges associated with the deployment of the robot manipulator for healthcare tasks, including compliant human-robot interaction, nonlinearity, model uncertainty and external disturbance, the UDE-based robust region tracking controller was developed. The UDE was integrated into the region reaching control framework with the backstepping technique. The proposed approach ensured the robot manipulator tracking the moving target trajectory with sufficient robustness in the presence of model uncertainty; furthermore, the compliance property for human-robot interaction was achieved with the regional feedback error. The effectiveness, robustness and compliant human-robot interaction capability of the developed control algorithm have been successfully validated through extensive experimental studies with a UR 10 robot manipulator, including region trajectory tacking and temperature measurement tasks.

\section{ACKNOWLEGMENT}

The work has been financially supported by National Natural Science Foundation of China (Grant Nos. 51805449 and 6210022036) and 1.3.5 project for disciplines of excellence, West China Hospital, Sichuan University (Grant Nos. ZYYC21004 and ZYJC21081)

\section{REFERENCES}

[1] G.-Z. Yang, B. J. Nelson, R. R. Murphy, H. Choset, H. Christensen, S. H. Collins, P. Dario, K. Goldberg, K. Ikuta, N. Jacobstein, D. Kragic, R. H. Taylor, and M. McNutt, "Combating COVID-19-the role of robotics in managing public health and infectious diseases," Science Robotics, vol. 5, no. 40, May 2020.

[2] WHO coronavirus disease (COVID-19) dashboard. (Accessed 2021, January 27). [Online]. Available: https://covid19.who.int/

[3] The Lancet, "COVID-19: protecting health-care workers," The Lancet, vol. 395, no. 10228, p. 922, Mar. 2020.

[4] Z. Li, P. Moran, Q. Dong, R. J. Shaw, and K. Hauser, "Development of a tele-nursing mobile manipulator for remote care-giving in quarantine areas," in Proceedings of IEEE International Conference on Robotics and Automation, 2017, pp. 3581-3586.

[5] G. Yang, H. Lv, Z. Zhang, L. Yang, J. Deng, S. You, J. Du, and H. Yang, "Keep healthcare workers safe: Application of teleoperated robot in isolation ward for COVID-19 prevention and control," Chinese Journal of Mechanical Engineering, vol. 33, no. 47, pp. 1-4, Jun. 2020.

[6] L. Robotics. Lifeline robotics swab robot. (Accessed 2021, January 27). [Online]. Available: https://www.lifelinerobotics.com/\#careebo-llr-s1

[7] S. Wang, K. Wang, R. Tang, J. Qiao, H. Liu, and Z.-G. Hou, "Design of a low-cost miniature robot to assist the COVID-19 Nasopharyngeal swab sampling," IEEE Transactions on Medical Robotics and Bionics, vol. 3, no. 1, pp. 289-293, Feb. 2021. 
[8] S. Wu, K. Li, R. Ye, Y. Lu, J. Xu, L. Xiong, A. Cui, Y. Li, C. Peng, and F. Lv, "Robot-assisted teleultrasound assessment of cardiopulmonary function on a patient with confirmed COVID-19 in a cabin hospital," Advanced Ultrasound in Diagnosis and Therapy, vol. 4, no. 2, pp. 128130, Jun. 2020.

[9] W. E. Dixon, "Adaptive regulation of amplitude limited robot manipulators with uncertain kinematics and dynamics," IEEE Transactions on Automatic Control, vol. 52, no. 3, pp. 488-493, Mar. 2007.

[10] D. Chen, S. Li, W. Li, and Q. Wu, "A multi-level simultaneous minimization scheme applied to jerk-bounded redundant robot manipulators," IEEE Transactions on Automation Science and Engineering, vol. 17, no. 1, pp. 463-474, Jan. 2020.

[11] Y. Huang, Y. S. Yong, R. Chiba, T. Arai, T. Ueyama, and J. Ota, "Kinematic control with singularity avoidance for teaching-playback robot manipulator system," IEEE Transactions on Automation Science and Engineering, vol. 13, no. 2, pp. 729-742, Apr. 2016.

[12] M. Jin, S. H. Kang, P. H. Chang, and J. Lee, "Robust control of robot manipulators using inclusive and enhanced time delay control," IEEE/ASME Transactions on Mechatronics, vol. 22, no. 5, pp. 21412152, Jun. 2017.

[13] H. Wang, "Adaptive control of robot manipulators with uncertain kinematics and dynamics," IEEE Transactions on Automatic Control, vol. 62, no. 2, pp. 948-954, Feb 2017.

[14] C. C. Cheah, S. Kawamura, and S. Arimoto, "Feedback control for robotic manipulator with an uncertain jacobian matrix," Journal of Robotic Systems, vol. 16, no. 2, pp. 119-134, Feb. 1999.

[15] C. C. Cheah, C. Liu, and J. J. E. Slotine, "Adaptive tracking control for robots with unknown kinematic and dynamic properties," The International Journal of Robotics Research, vol. 25, no. 3, pp. 283296, Mar. 2006

[16] C. C. Cheah and H. C. Liaw, "Inverse jacobian regulator with gravity compensation: stability and experiment," IEEE Transactions on Robotics, vol. 21, no. 4, pp. 741-747, Aug. 2005.

[17] H. Wang, W. Ren, C. C. Cheah, Y. Xie, and S. Lyu, "Dynamic modularity approach to adaptive control of robotic systems with closed architecture," IEEE Transactions on Automatic Control, vol. 65, no. 6 , pp. 2760-2767, Jun. 2020.

[18] M. A. Arteaga-Perez, J. Pliego-Jimenez, and J. G. Romero, "Experimental results on the robust and adaptive control of robot manipulators without velocity measurements," IEEE Transactions on Control Systems Technology, vol. 28, no. 6, pp. 2770-2773, Oct. 2020

[19] J. Nubert, J. Kohler, V. Berenz, F. Allgower, and S. Trimpe, "Safe and fast tracking on a robot manipulator: Robust MPC and neural network control," IEEE Robotics and Automation Letters, vol. 5, no. 2, pp. 30503057, Apr. 2020.

[20] A. Ferrara and G. P. Incremona, "Design of an integral suboptimal second-order sliding mode controller for the robust motion control of robot manipulators," IEEE Transactions on Control Systems Technology, vol. 23, no. 6, pp. 2316-2325, Nov. 2015.

[21] J. Lee, P. H. Chang, and M. Jin, "An adaptive gain dynamics for time delay control improves accuracy and robustness to significant payload changes for robots," IEEE Transactions on Industrial Electronics, vol. 67, no. 4, pp. 3076-3085, Apr. 2020.

[22] Q.-C. Zhong and D. Rees, "Control of uncertain LTI systems based on an uncertainty and disturbance estimator," ASME Journal of Dynamic Systems, Measurement, and Control, vol. 126, no. 4, pp. 905-910, Mar. 2004.

[23] J. P. Kolhe, M. Shaheed, T. S. Chandar, and S. E. Taloe, "Robust control of robot manipulators based on uncertainty and diturbance estimation," International Journal of Robust and Nonlinear Control, vol. 23, no. 1, pp. 104-122, Oct. 2013.

[24] Y. Dong and B. Ren, "UDE-based variable impedance control of uncertain robot systems," IEEE Transactions on Systems, Man and Cybernetics: Systems, vol. 49, no. 12, pp. 2487-2498, Dec. 2019.

[25] Q. Lu, B. Ren, and S. Parameswaran, "Uncertainty and disturbance estimator-based global trajectory tracking control for a quadrotor," IEEE/ASME Transactions on Mechatronics, vol. 25, no. 3, pp. 15191530, Jun. 2020.
[26] B. Ren, J. Dai, and Q. Zhong, "UDE-based robust output feedback control with applications to a piezoelectric stage," IEEE Transactions on Industrial Electronics, vol. 67, no. 9, pp. 7819-7828, Sep. 2020.

[27] Y. Li, S. S. Ge, C. Yang, X. Li, and K. P. Tee, "Model-free impedance control for safe human-robot interaction," in Proceedings of IEEE International Conference on Robotics and Automation, 2011, pp. 60216026.

[28] X. Liu, S. S. Ge, F. Zhao, and X. Mei, "A dynamic behavior control framework for physical human-robot interaction," Journal of Intelligent \& Robotic Systems, vol. 101, no. 1, p. 14, Dec. 2020.

[29] J. Choi, J. Baek, W. Lee, Y. S. Lee, and S. Han, "Adaptive model-free control with nonsingular terminal sliding-mode for application to robot manipulators," IEEE Access, vol. 8, pp. 169897-169 907, Sep. 2020.

[30] J. W. Yu, X. H. Zhang, J. C. Ji, J. Y. Tian, and J. Zhou, "Region-Reaching Control of a Flexible-Joint Manipulator," ASME Journal of Dynamic Systems, Measurement, and Control, vol. 142, no. 11, p. 114503, Jul. 2020.

[31] C.-H. Lin, Y.-Y. Su, Y.-H. Lai, and C.-C. Lan, "A spatial-motion assistas-needed controller for the passive, active, and resistive robot-aided rehabilitation of the wrist," IEEE Access, vol. 8, pp. 133951-133960, Jul. 2020.

[32] K. P. Michmizos, S. Rossi, E. Castelli, P. Cappa, and H. I. Krebs, "Robot-aided neurorehabilitation: A pediatric robot for ankle rehabilitation," IEEE Transactions on Neural Systems and Rehabilitation Engineering, vol. 23, no. 6, pp. 1056-1067, Mar. 2015.

[33] M. Hagenow, E. Senft, R. Radwin, M. Gleicher, B. Mutlu, and M. Zinn, "Informing real-time corrections in corrective shared autonomy through expert demonstrations," IEEE Robotics and Automation Letters, vol. 6, no. 4, pp. 6442-6449, Oct. 2021.

[34] M. Marcano, S. Díaz, J. Pérez, and E. Irigoyen, "A review of shared control for automated vehicles: Theory and applications," IEEE Transactions on Human-Machine Systems, vol. 50, no. 6, pp. 475-491, Dec. 2020.

[35] C. C. Cheah, D. Q. Wang, and Y. C. Sun, "Region-reaching control of robots," IEEE Transactions on Robotics, vol. 23, no. 6, pp. 1260-1264, Dec. 2007.

[36] S. P. Hou and C. C. Cheah, "Region tracking control for robot manipulators," in Proceedings of IEEE International Conference on Control Applications, 2007, pp. 1438-1443.

[37] X. Li and C. C. Cheah, "Adaptive regional feedback control of robotic manipulator with uncertain kinematics and depth information," in Proceedings of American Control Conference, 2012, pp. 5472-5477.

[38] X. Li, G. Chi, S. Vidas, and C. C. Cheah, "Human-guided robotic comanipulation: Two illustrative scenarios," IEEE Transactions on Control Systems Technology, vol. 24, no. 5, pp. 1751-1763, Sep. 2016.

[39] W. He, C. Xue, X. Yu, Z. Li, and C. Yang, "Admittance-based controller design for physical human-robot interaction in the constrained task space," IEEE Transactions on Automation Science and Engineering, vol. 17, no. 4, pp. 1937-1949, Oct. 2020.

[40] M. Xie, Y. Wang, G. Feng, and D. Sun, "Automated pairing manipulation of biological cells with a robot-tweezers manipulation system," IEEE/ASME Transactions on Mechatronics, vol. 20, no. 5, pp. 22422251, Oct. 2015.

[41] S. S. Ge and Y. J. Cui, "New potential functions for mobile robot path planning," IEEE Transactions on Robotics and Automation, vol. 16, no. 5, pp. 615-620, Oct. 2000.

[42] S. S. Ge and C.-H. Fua, "Queues and artificial potential trenches for multirobot formations," IEEE Transactions on Robotics, vol. 21, no. 4, pp. 646-656, Aug. 2005.

[43] B. Ren, S. S. Ge, T. H. Lee, and M. Krstic, "Region tracking control for multi-agent systems with high-order dynamics," in Proceedings of American Control Conference, 2013, pp. 1266-1271.

[44] B. Ren, Q.-C. Zhong, and J. Dai, "Asymptotic reference tracking and disturbance rejection of UDE-based robust control," IEEE Transactions on Industrial Electronics, vol. 64, no. 4, pp. 3166-3176, Apr. 2017.

[45] M. Krstic, P. V. Kokotovic, and I. Kanellakopoulos, Nonlinear and adaptive control design. John Wiley \& Sons, Inc., 1995.

[46] S. Lyu and C. C. Cheah, "Human-robot interaction control based on a general energy shaping method," IEEE Transactions on Control Systems Technology, vol. 28, no. 6, pp. 2445-2460, Nov. 2020. 